\title{
AN INDIRECT BOUNDARY ELEMENT METHOD FOR 2-D FINITE/INFINITE REGIONS WITH MULTIPLE DISPLACEMENT DISCONTINUITIES
}

\author{
NABIL FARES and VICTOR C. LI \\ Department of Civil Engineering, Massachusetts Institute of Technology, Cambridge, \\ MA 01239, U.S.A.
}

\begin{abstract}
An indirect boundary element method based on the superposition of the elastic fields of edge dislocations is derived. Elements having an arbitrarily specified distribution of dislocations is made possible through the use of a specialized numerical integration scheme. The method is applied to finite as well as infinite regions with single or multiple cracks. Sample results indicate that the method is well suited for multiply cracked problems in infinite regions, whereas a large number of degrees of freedom is required for the accurate solution of multiply cracked problems in finite regions. Implementation of the method to nonlinear boundary conditions is discussed. To demonstrate the applicability of the numerical method to nonlinear boundary condition problems, an analysis of the propagation of part-through crack problems in plates under in-plane loadings is performed through the use of the line spring technique and the present numerical method.
\end{abstract}

\section{INTRODUCTION}

FRACTURE problems characteristically have localized regions of high stress and strain field gradients. This feature makes crack problems "expensive" to solve using the finite element method (FEM) and hence researchers have naturally turned to alternatives in numerical approaches to fracture problems. The "expense" in using finite elements comes about because a lot of elements are needed to capture the elastic fields around the cracktips accurately, although this problem is somewhat reduced by the introduction of special crack tip elements. Furthermore, crack propagation requires remeshing at every propagation step since the cracktip location is continuously changing. This feature makes regular FEM analysis of cracked structures undesirable.

The displacement discontinuity (DD) method as originated by Crouch[1] combined the idea of modelling cracks as distributions of dislocations with the integral equation methods. Crouch used the DD method to solve crack problems in infinite space and non-crack problems in finite space (both in ref. [1]).

The integral equation methods were later refined by Brebbia et al.[2], and later by other researchers (e.g. Banerjee and Butterfield[3]) into an efficient and versatile numerical algorithm for solving linear elastic solid mechanics problems called the Boundary Element Method (BEM). The Green's functions used in the boundary element methods were fundamental point load solutions in infinite space (Kelvin's solution) and this indirect boundary element method was essentially the DD method except for the change in Green's functions.

The use of the direct boundary element method by Blandford et al.[4] to model problems of cracks in finite regions was partially successful. A severe shortcoming was the inefficiency of dealing with more than one crack in a finite region and the inability to handle crack problems in infinite regions.

Meanwhile, the modelling of crack problems as distributions of dislocations was refined by Crawford and Curran [5], and later by Curran and Vandamme[6] using the DD method as well as by Annigeri and Cleary[7] using a surface integral method. The surface integral method (SI) consisted of using Chebyshev numerical integration along the line of the crack with the dislocation density being the unknown parameters and hence implicitly assuming that the variation of the discontinuity is of the form of polynomials multiplied by an ellipse having as end points the tips of the crack. The shortcoming of the DD approach was the limitation in the allowable shapes of the distribution of dislocations and this was due to the limitations imposed by the analytic derivation of discontinuity elements. The shortcoming of the SI approach was both in the implicit assumptions of the shapes of the distribution of discontinuities (essentially polynomials multiplied by ellipses) and in the restrictions in the type of boundary conditions that can be imposed. Both the DD approach and the SI approach were used to solve problems in infinite regions. 
A major breakthrough was achieved by Annigeri and Cleary[7], by the hybridization of the SI technique with the finite element method. The method essentially consisted of subtracting the singularity generated by the cracks from the finite element scheme and then adding it back (in a consistent manner) via the SI scheme, allowing the finite element method to focus on the non-singular fields only.

This paper presents the following:

(i) The extension of the modelling of cracks by distribution of dislocations to include any arbitrary specified shapes (this is achieved by a specialized numerical integration technique). The method is used to model cracks in infinite as well as in finite regions by using the DD elements to model the outer boundaries as well as the cracks.

(ii) A general procedure for linear and nonlinear boundary conditions is formulated. The formulation is specialized to line spring modelling techniques for the propagation of part through cracks in plates under in-plane loading. Line spring models originated with Rice and Levy[8].

\section{GENERAL FEATURES OF THIS INDIRECT BOUNDARY ELEMENT METHOD}

The numerical method discussed in this paper can be formally classified as an indirect boundary element method. It is a boundary element method because only the boundaries of the region under consideration (and the discontinuity lines within the region) need to be discretized and not the whole region under consideration as in the finite difference and finite element methods. It is an indirect boundary element method because intermediate variables have to be calculated before the stress and displacement fields can be computed. The terminology of discontinuity lines instead of "cracks" is used in order to emphasize that the stress fields at the tips of the discontinuity lines need not be the $\mathrm{K}$ dominated fields that are familiar in linear elastic fracture mechanics, but may in fact involve no singularities or perhaps a "severe" singularity of $1 / r$ which corresponds to a dislocation tip. The choice of the strength of singularity depends on the particular physical problem under consideration.

A more descriptive term for the numerical method discussed in this paper would be a superposition method. The numerical method is built up using the following steps:

(a) A fundamental solution, in this case a dislocation dipole, is integrated over a line segment with the "shape" of the distribution of dislocations along the line segment being controlled by a few parameters. (The dislocation modes usually come in pairs in 2-D elasticity, corresponding to the "opening" and "sliding" dislocations.) This line segment is then called an element. The stress field of an element is everywhere in equilibrium, being constructed by the integration of stress fields that are in equilibrium. The displacement field of an element is everywhere compatible except along the line segment over which the element is defined; along that line segment, the displacement field has a discontinuity whose variation is controlled by the same parameters that control the distribution of dislocations along the same line segment.

(b) The boundary of the problem as well as all discontinuity lines is traced using these elements (see Fig. 1), and the solution of the elasticity problem under consideration is assumed (or approximated) to be the sum of the elastic fields of all the elements (hence the term superposition).

(c) The boundary conditions (imposed on the boundaries as well as on the discontinuity lines) are only satisfied at discrete points of the boundaries and discontinuity lines (this is usually called a

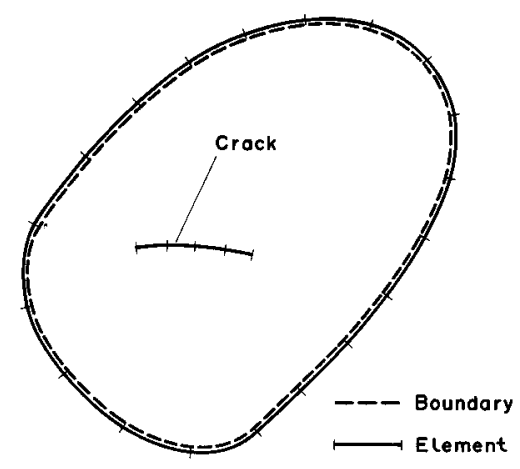

Fig. 1. The boundary of the region to be studied is discretized using elements placed slightly outside the boundary. The crack line is discretized using elements placed on the crack. 
collocation procedure). "Enough" collocation points for the boundary conditions are chosen in order for the parameters controlling the dislocation distributions to be determined. Using these parameters the stress and displacement fields throughout the region are determined.

The convergence of the indirect boundary element is not guaranteed as the number of elements increases indefinitely (convergence of collocation methods have been proven for some partial differential equation operators and expansion functions, when the collocation points were chosen in a specific manner). However, the system of equations formed using this indirect boundary element method is well conditioned and strongly diagonally dominant when the elements are of equal sizes, the curvature of the boundary is low, and the number of elements per unit arc length is not too "large". The upper bound for the number of elements per unit arc length that can be used depends (for a given type and grading of elements and a given geometry) on the arithmetic accuracy of the machine being used, and would have to be determined empirically. For example, for double precision accuracy on a Vax 11-750, the upper bound for the number of "constant" elements with uniform sizes for a planar crack per unit arc length is at least above 50 elements.

The well conditioning of the system of equations formed is due to the dislocation singularities at the tips of each element causing the value of the line integrals evaluated at field points within the element to have magnitudes higher than for field points on adjacent elements. That is why it is advisable to trace the boundary of the region under consideration with the elements and not to place the elements too far away from the boundaries. Note, however, that in all cases the collocation points have to be chosen at the boundaries.

The above discussion on the conditioning of the equations is dependent on the type of singularity used to construct the elements. For example, for the "classical" boundary element method (using point forces, Kelvin's solution) the conditioning of the equations is expected to be worse (although still sufficient) than for the dislocation based boundary element method (since the integrated terms would contain (in 2-D problems) " $\log r$ " terms for the point force based method vs " $1 / r$ " terms for the dislocation based method). Note, however, that the point force based elements for the outer boundaries approximate more accurately applied traction boundary conditions than dislocation based elements, and hence would lead to more accurate results close to the boundaries (see section on sample results, and the conclusion for further remarks).

Finally, the method is very good at capturing the elastic fields due to discontinuity lines, because it is built up using the fundamental solutions that are required to describe such fields (i.e. dislocation dipole fields from which a line of discontinuity of any variation in the discontinuity can be constructed). However, due to the use of singularities at the boundaries, the solution of the problem using this numerical method is expected to have problems close to the boundaries (see the section on sample problems).

The next two sections will discuss the technical details related to the generation of elements and the specifications of boundary conditions.

\section{GENERATION OF ELEMENTS}

The generation of elements requires the analytic or numerical evaluation of a line integral. This line integral has an integrand which is singular when the evaluation point lies on the line of integration and the line integral is then only defined in the principal value sense. We have:

$$
\begin{aligned}
\sigma_{i j}(\mathbf{x}) & =\int_{L} G_{i j}^{\mathrm{1}}(\mathbf{x}, s) M(s) \mathrm{d} s+\text { "tip effects" } \\
u_{j}(\mathbf{x}) & =\int_{L} G_{j}^{2}(\mathbf{x}, s) M(s) \mathrm{d} s+\text { "tip effects" }
\end{aligned}
$$

where: $L$ is the line segment considered (the elements), $\mathbf{x}$ any arbitrary point of interest (a field point), $s$ the local coordinate along the element, $G_{i j}^{1}$ the dislocation fundamental solution for the stress field, $G_{j}^{2}$ the dislocation fundamental solution for the displacement field, and $M(s)$ the variation of the dislocation slopes along the line segment. For the generality of formulation of boundary conditions (BCs), it is desirable to directly control the dislocation "shapes" instead of controlling the "shapes" of the dislocation slopes. One way to achieve that is by integrating the above equation by parts; we get: 


$$
\begin{aligned}
& \sigma_{i j}(\mathbf{x})=\left.G_{j}^{1}(\mathbf{x}, s)\left[N(s)-N\left(s^{*}\right)\right]\right|_{s=0^{+}} ^{s=l^{-}}-\int_{L} G_{j}^{3}(\mathbf{x}, s)\left[N(s)-N\left(s^{*}\right)\right] \mathrm{d} s+\text { "tip effects" } \\
& u_{j}(\mathbf{x})=\left.G_{j}^{2}(\mathbf{x}, s)\left[(s)-N\left(s^{*}\right)\right]\right|_{s=0^{+}} ^{s=l^{-}}-\int G_{i j}^{4}(\mathbf{x}, s)\left[N(s)-N\left(s^{*}\right)\right] \mathrm{d} s+\text { "tip effects" }
\end{aligned}
$$

where $G_{i j}^{3}$ is the dislocation dipole fundamental solution for the stress field, $G_{j}^{4}$ the dislocation dipole fundamental solution for the displacement field, $N(s)$ the variation of the dislocation along the line segment, and $s^{*}$ a constant value to be discussed below. The first terms are to be evaluated between $s=l^{-}$, and $s=0^{+}$. This is particularly relevant for $N(s)$ because the dislocation values along the line segment might have discontinuities after passing the tips.

Note that the above formulation can be generalized to 3-D, whereas the previous one cannot, due to the 2-D nature of a dislocation fundamental solution.

There are two points to be clarified in the above formulation. First, what are the "tip effects"? Second, the introduction of the term $N\left(s^{*}\right)$ and the value of $s^{*}$.

When a variation of dislocations along a line segment is chosen, the variation might have nonzero values at the tips of the line segment. We could either choose to "suddenly" bring the dislocation values at the tips to zero (this is done by placing negative dislocations of appropriate magnitudes at the tips of the line segments) or we could allow the dislocation value to maintain its magnitude proceeding to infinity (the dislocation will proceed in a straight line tangent to the slope of the line segment from the concerned tip). Placing negative dislocations at the tips of the elements in order to "close" the discontinuity value (when it is non-zero) is what is meant by "tip effects".

For example the dislocation value within the "constant" element formulated by Crouch[1] is constant within the element, but has "jumps" in the dislocation when passing the tips; therefore, for the constant element, the stress field is wholly due to the "tip effects", i.e. to whether we have any "jumps" in the dislocation value at the tips. In general, the stress field generated by an element is due to either "jumps" in the dislocation values at the tips ("tip" effects) or due to gradients in the dislocation values within the element.

The dislocation dipole stress field is highly singular with singularity of order $O\left(1 / r^{2}\right)$. This makes the line integral for the stress field undefined when the field point lies on the element, unless if the dislocation dipole is multiplied with a function that vanishes at least as fast as $O(r)$ at the field point. If the function vanishes exactly as $O(r)$ then we still have to evaluate a PV integral.

In order to make the line integral well defined, the value of $s^{*}$ is chosen so that $N(s)-N\left(s^{*}\right)=0$ when " $s$ " coincides with a field point that lies on the element. In order to make the line integral numerically accurate for low integration orders as a field point approaches the element the value of $s^{*}$ is chosen to be the orthogonal projection of the field point on the line segment with respect to the "local normal coordinate" of the element.

In the case when $s^{*}$ lies within the element, numerical integration is performed by "cutting" the line segment into two intervals (see Fig. 2). The first interval is chosen in such a way that $s^{*}$ is at the center of that segment; an even-order Gauss-Legendre integration is performed on that line segment to approximate a PV integral and to avoid evaluating the singular part of the integrand (the Green's function) at the singularity point. A line integral over the second interval is then calculated (also using

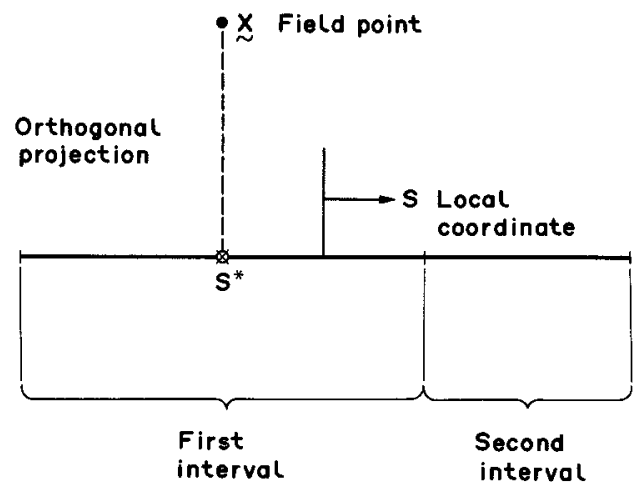

Fig. 2. An element is subdivided into two intervals for the purpose of the numerical integration scheme. The first interval "centers" the field point (see text for details). 
Gauss-Legendre integration) and the result added to the PV integral over the first interval to obtain the final result.

Why the insistence on numerical integration?

First, a wider choice of elements is helpful. For example, choosing elements that have their discontinuities varying in the form of polynomials multiplied by an elliptic "shape" is much more efficient at modelling Griffith cracks than piecewise parabolic elements with crack tip elements at each end. In fact, if the prevailing stress field around the crack is in the form of a polynomial, an exact solution can be obtained. The use of elliptic shapes multiplied by polynomials for the dislocation discontinuities in order to model crack problem has been used by Kachanov [9], but with a different approach from collocation to determine the unknown parameters.

Second, the asymptotic stress field at an element's tips can be much better controlled. These stress fields can range from having $(1 / r)$ singularities, to the $(1 / \sqrt{r})$ "K-like" crack tips to a "no singularity" crack tip. The last type can be useful for analyzing cohesive zone models such as those proposed by Barenblatt[10].

Finally, some elements are computationally less efficient to calculate with a closed form solution

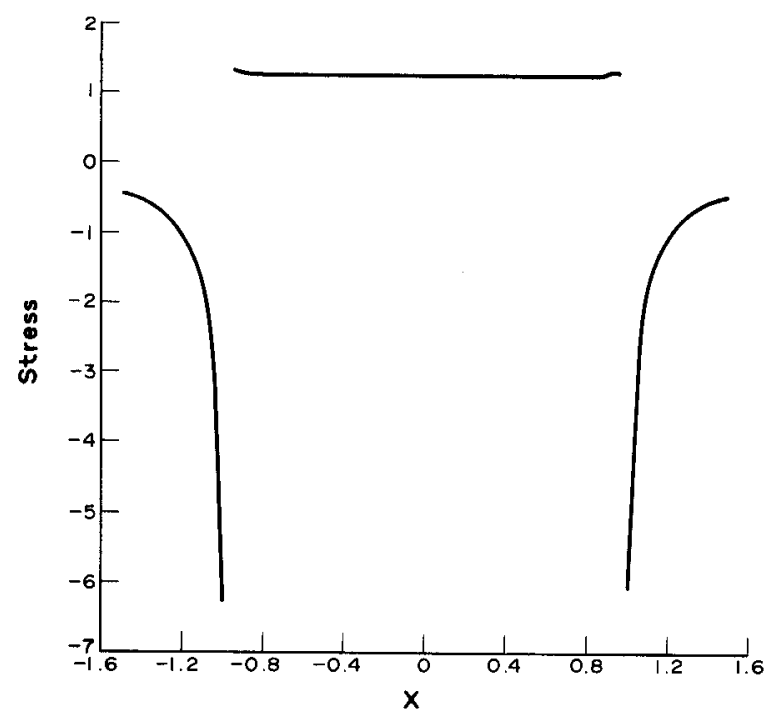

Fig. 3. A uniformly pressurized crack can be modelled exactly using an "ellipse" element placed at $(-1.0$ $<x<1.0$ ), since it exactly satisfies the constant stress boundary condition on the crack.

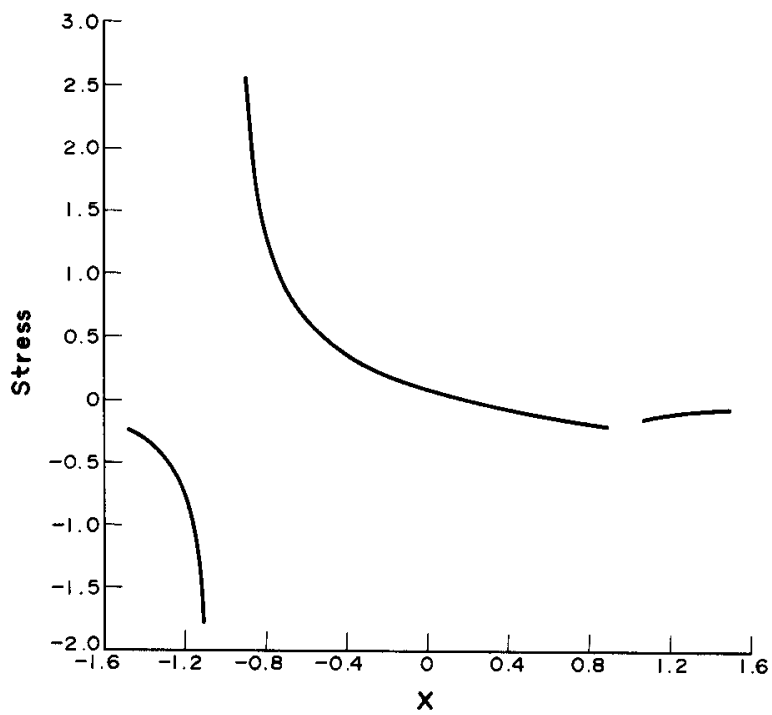

Fig. 4. An $r^{3 / 2}$ opening discontinuity at the right tip $(x=1.0)$ leads to a finite stress field. The $1 / r$ singularity at the left tip $(x=-1.0)$ is associated with a dislocation. The stress scales with the imposed opening at any fixed point on the element. 


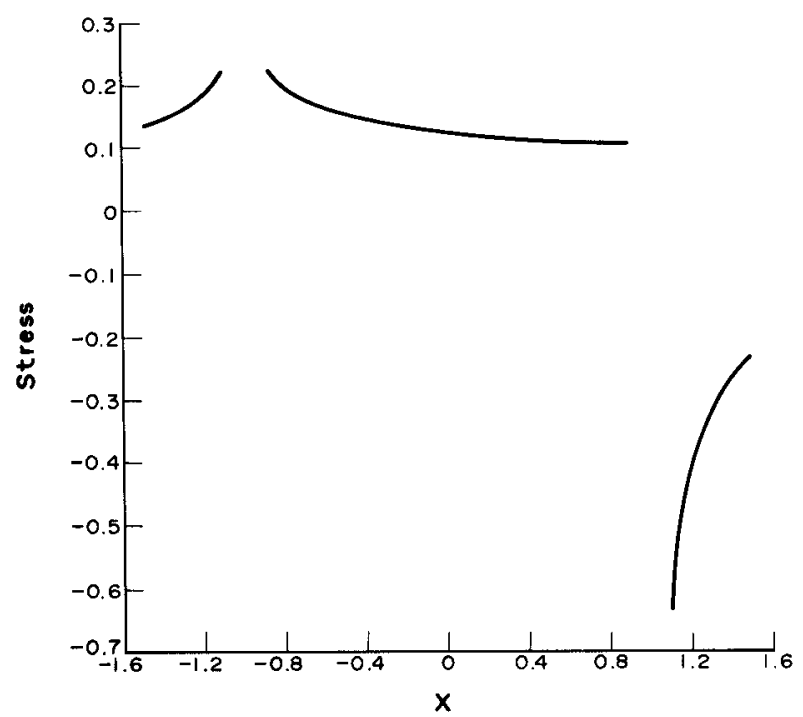

Fig. 5. A $\sqrt{r}$ opening discontinuity at the right tip leads to a nearly constant stress field within the element, and a $1 / \sqrt{r} \mathrm{~K}$-like stress field outside the element close to the right tip.

(which sometimes involve very complex functions) than by numerical integration (which usually only requires the evaluation of algebraic and square root terms). For example this might be the case for the crack tip elements developed by Curran and Vandamme[6].

Examples of the stress fields of some elements extending from $x=-1$ to +1 at field points that are on the discontinuity line $y=0$ are shown.

In Fig. 3, a simple elliptic shape for the variation of the dislocation over the element gives the stress field of a Griffith crack under constant pressure.

In Fig. 4, a zero slope crack tip at $x=+1$ gives a bounded stress field (within the resolution of the plot) at that tip, whereas, the tip $x=-1$ corresponds to a dislocation tip.

In Fig. 5, a "K-like" crack tip element has the $1 / \sqrt{r}$ singularity at $x=+1$ whereas the tip at $x=-1$ corresponds to a dislocation that is allowed to continue to infinity (i.e. the "tip effects" mentioned earlier is not included).

In Figs 3-5 stress values in a small interval at each of the tips $x=-1$ and $x=1$ have been omitted either due to loss of accuracy in the numerical results or due to the existence of singularities at some of the tips.

\section{SPECIFICATION OF BOUNDARY CONDITIONS}

A general form for the specification of the boundary conditions is chosen as follows:

$$
\alpha_{1} T_{n}+\alpha_{2} T_{t}+\beta_{1} u_{n}+\beta_{2} u_{t}=\gamma
$$

where $T_{n}$ is the normal component of the traction along the boundary, $T_{t}$ the tangential component of the traction along the boundary, $u_{n}$ the normal component of the displacement along the boundary or dislocation along a discontinuity line, and $u_{t}$ the tangential component of the displacement along the boundary or the dislocation along a discontinuity line. The $\alpha$ values, $\beta$ values and $\gamma$ values can either be constants or functions of any traction, displacement or dislocation component.

The above formation can be used to model spring conditions (e.g. $\alpha_{2}=\beta_{2}=\gamma=0, \alpha_{1}=1$ and $\beta_{1}=$ negative the spring stiffness for a spring condition normal to the boundary or discontinuity line), as well as friction (e.g. $\beta_{1}=\beta_{2}=\gamma=0, \alpha_{1}=1$ and $\alpha_{2}=$ negative the coefficient of friction).

When nonlinear effects in the medium occur (e.g. due to non-linear material behavior or due to large displacements and/or large strains), the principle of superposition method is no longer applicable and the method described in this paper can no longer be used. However, when the boundary conditions are nonlinear, our superposition method is still valid, provided we satisfy the nonlinear boundary conditions. Nonlinear boundary conditions are also satisfied at discrete points 
along the boundaries (i.e. collocated). However, the collocation procedure gives us a set of nonlinear equations in terms of the parameters controlling the shapes of the dislocation discontinuity in the elements.

Several methods for the solution of simultaneous nonlinear equations are known in the literature, e.g. the Newton-Raphson method and the secant method. Each method has certain advantages and certain disadvantages, ranging from "ease of programming" to rates and conditions for convergence; however, what they all have in common is that an iteration procedure is required.

The contribution factor of any given parameter (controlling the shape of the variation of dislocations within a given element) to the stress or displacement field at a collocation point is calculated by appropriate line integrals (see previous section). We note that, during an iteration (corresponding to a given solution method), the contribution factors do not change, since the medium is linear. However, nonlinear functions of the tractions and displacements (and hence of the contribution factors), has to be updated at each iteration step. Therefore, the contribution factors have to be calculated and stored before the iteration procedure starts in order to avoid recalculating the line integrals that determine those factors at each iteration step.

The secant method was successfully implemented for the nonlinear boundary condition encountered in the propagation of part-through cracks modelled by the line spring technique.

\section{SAMPLE PROBLEMS}

The method outlined above has been used to calculate stress intensity factors for some sample problems in finite and infinite space. In addition, the propagation of part-through cracks in plates using the line spring model has been implemented using this method.

Before presenting stress intensity results for some sample crack problems, an illustrative problem showing the characteristics of this method with finite boundaries will be presented.

A cantilever problem [having no cracks, Fig. 6(a) insert] has been solved using this boundary element method. The parameters of the problem were chosen the same as in [5] and are shown in Fig. 6(a). This example problem is repeated in order to make the following comments.

The shear and bending stress distribution is plotted for $x=1.87$ and is compared with the Bernoulli-beam solution [Fig. 6(a) and (b)]. Relatively large errors are encountered close to the boundaries. These errors are implicit to the numerical method and are not due to numerical inaccuracies caused by numerical integrations, roundoff errors or truncation errors but are solely due to the superposition of singular fields to numerically solve a problem that is expected to yield "regularly" varying fields. These errors are much less severe if point force based elements are used instead, since the singularity of point force based elements is much less severe.

The calculated vertical displacements at the centerline of the beam are shown in Fig. 6(c), and are compared with the Timoshenko-beam solution, ref. [11]. The accuracy of those displacements does not deteriorate near the boundaries, indicating that the fields are at most weakly singular at the element tips that trace the boundaries.

Stress intensity factors for some representative problems are presented next. The stress intensity factors are calculated for a given crack tip using the following formula and by taking the limit numerically:

$$
K=\lim _{r \rightarrow 0} M \delta(r) / \sqrt{r}
$$

where $M$ is a modulus discussed below, $r$ the distance from the crack tip (in the crack plane), $K$ the stress intensity factor (mode I or II), and $\delta$ the crack tip opening. " $M$ " is a modulus that depends on the material properties as well as whether the problem is a plane strain or plane stress problem. The value of $M$ is:

$$
\begin{array}{ll}
M=G \sqrt{2 \pi} /[4(1-v)] & \text { (plane strain) } \\
M=G(1+v) \sqrt{2 \pi} / 4 & \text { (plane stress) }
\end{array}
$$

where $G$ is the shear modulus and $v$ Poisson's ratio. Note that the type of elements to which the above 

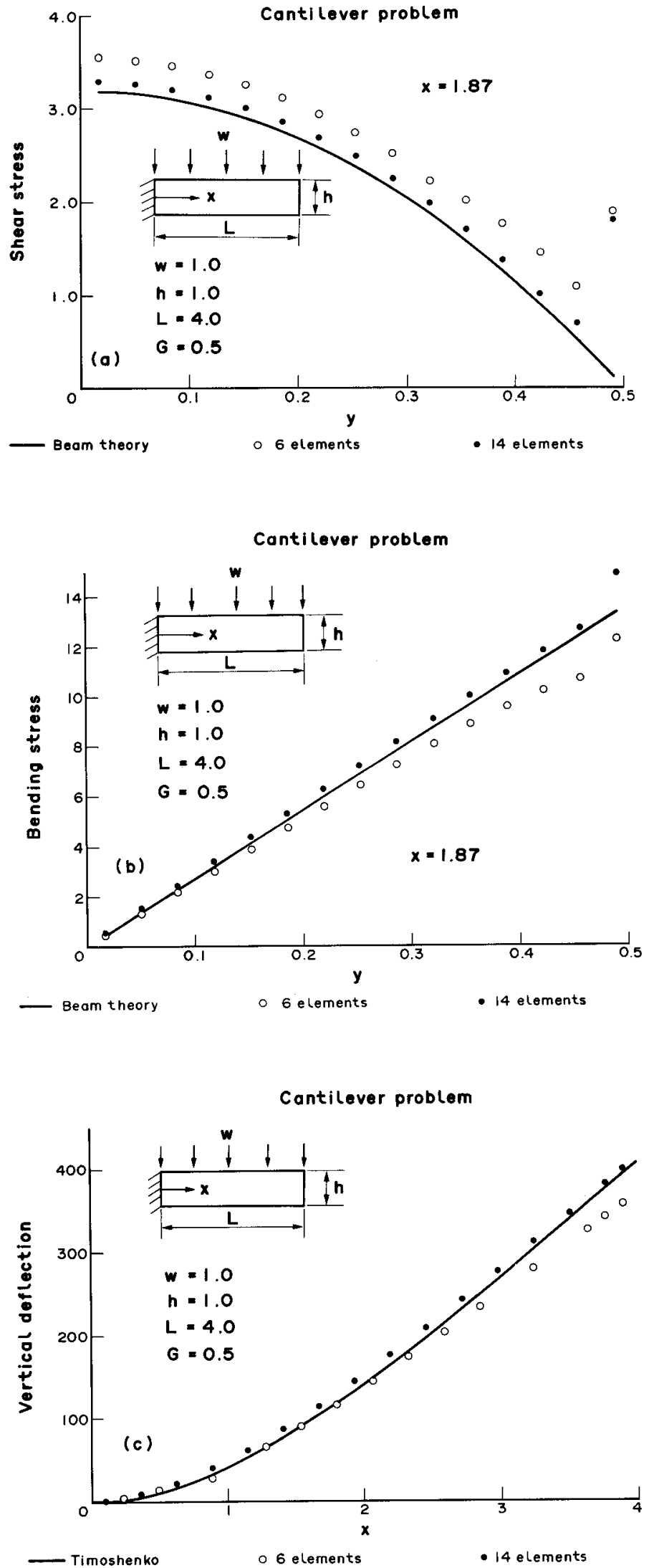

Fig. 6. A cantilever beam under uniform load is discretized using six and 14 parabolic elements. (a) and (b) The shearing and bending stresses respectively show relatively large errors close to the boundary. (c) The vertical displacements along the centerline are compared with the "Timoshenko beam" solution. 
formula for $K$ can be applied have to contain a $\sqrt{r}$ asymptotic opening behavior as the tip is approached (e.g. the "ellipse" element and the crack tip element with the $\sqrt{r}$ plus higher-order terms). For those elements, it was found that the stress intensity factor calculations are relatively insensitive to how small the value of " $r$ " is taken in the limit as long as it is much smaller (e.g. by two orders of magnitude) than the size of the element under consideration.

Results for the following configurations are presented:

(i) staggered cracks in infinite space [Fig. 7(a) and (b)],

(ii) star crack configuration in infinite space (Fig. 8, Table 1),

(iii) center-cracked finite size panel (Fig. 9, Table 2), and

(iv) side-cracked finite size panel (Fig. 10, Table 3).

The discretization of the above configurations is as follows:

(i) Two quadratic-elliptic elements were used for $(t / a)>0$ [see Fig. 7(a)]. For values of $(t / a)>0$ the quadratic-elliptic elements were found to be inadequate, the five parabolic (i.e. dislocations are varying as a quadratic polynomial over an element) plus two two-parameter $\left(1 / \sqrt{r}\right.$ and $1 / r^{3 / 2}$ terms) crack tip elements for each crack were used instead. The results are in good agreement with the semianalytic results (Yokobori et al. [12]), except for the $\mathrm{K}_{\mathrm{II}}$ values at $(t / a)=-0.5$ and 0.0 , where a higher discretization is required for accurate results to be obtained. The loss of accuracy (for the same
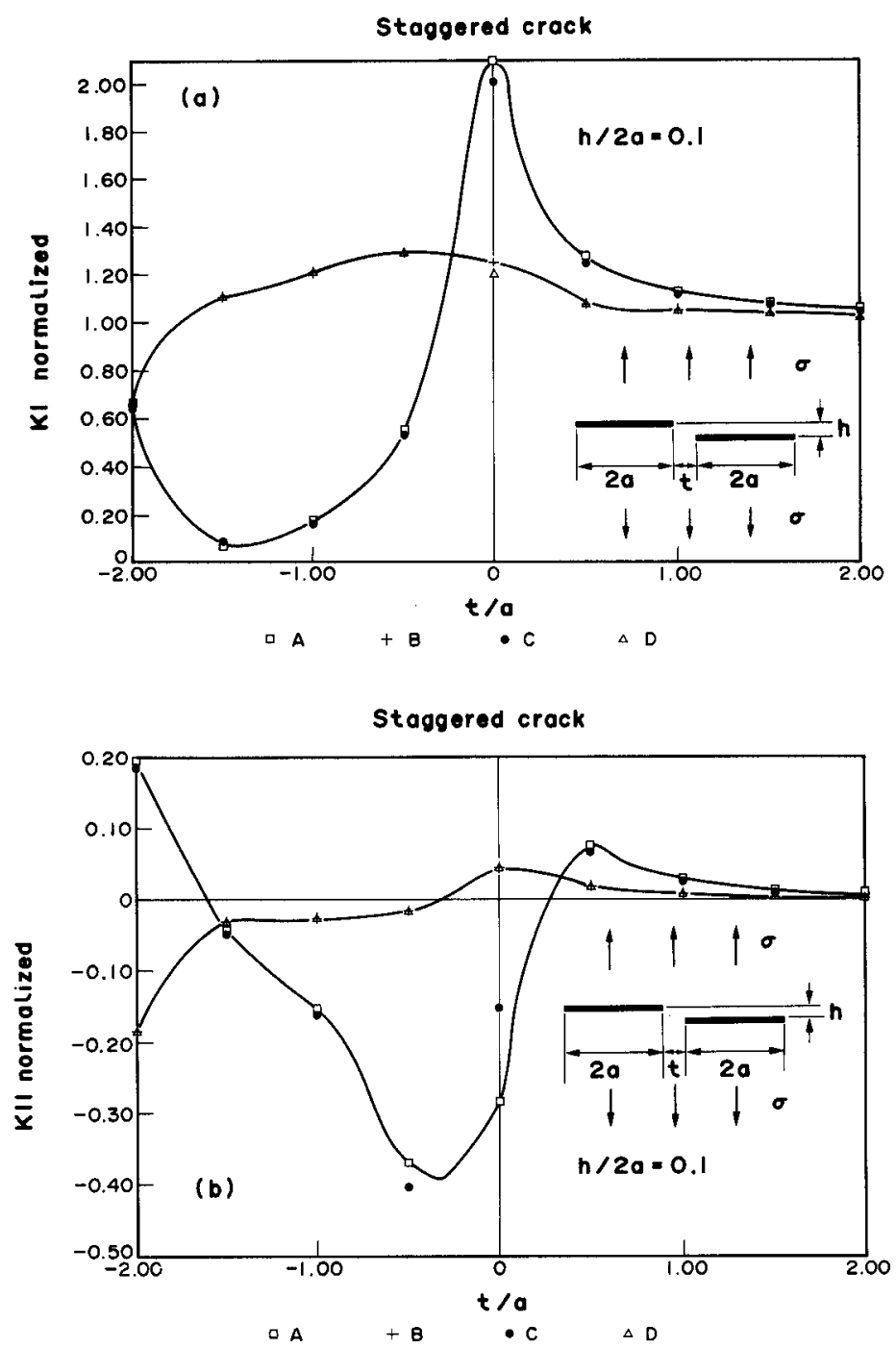

Fig. 7. Stress intensity factors in mode I (a) and in mode II (b) are calculated using the present numerical scheme. When compared with the analytic solution, the results indicate a need for a higher discretization when the tips are close and overlapping ( $A$ and $B$ are the analytic results, $C$ and $D$ are the calculated results at the inner and outer tips respectively). 


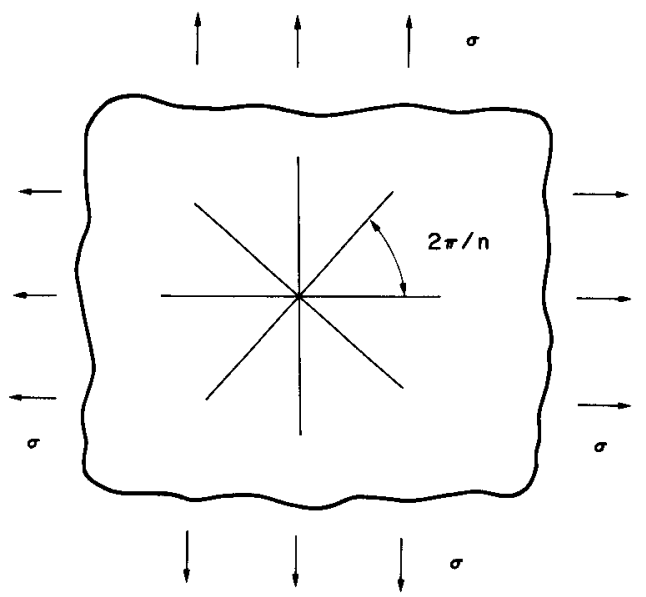

Fig. 8. The geometry of a star crack under hydrostatic far field stress.

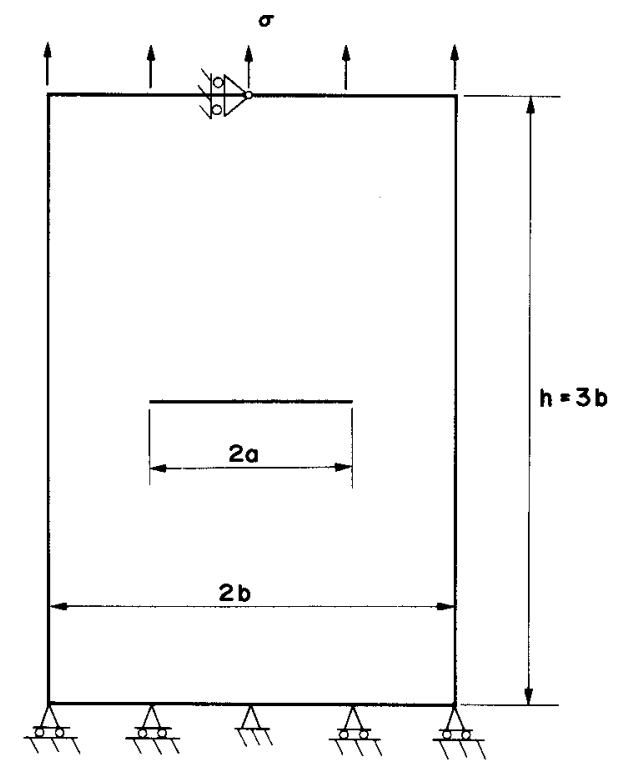

Fig. 9. Geometry of a center cracked panel and boundary conditions imposed.

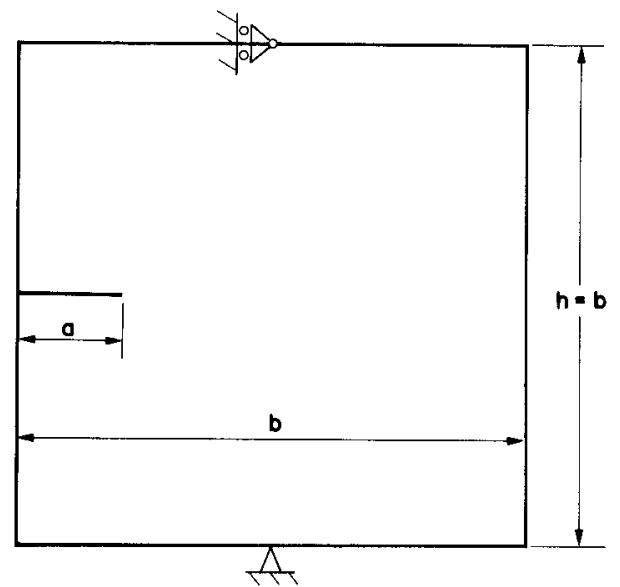

Fig. 10. A side cracked panel is uniformly pressurized and is constrained from rigid body motions by two hinges placed at the locations shown. 
Table 1. Comparison of stress intensity factors for a star crack

\begin{tabular}{rccc}
\hline$n$ & $\begin{array}{c}\text { Ref. }[13] \\
F(n)\end{array}$ & $\begin{array}{c}\text { Calculated } \\
F(n)\end{array}$ & $\%$ Error \\
\hline 4 & 0.864 & 0.8666 & 0.3 \\
6 & 0.754 & 0.7441 & -1.3 \\
8 & 0.677 & 0.6576 & -2.9 \\
10 & 0.615 & 0.5926 & -3.6 \\
\hline
\end{tabular}

Table 2. Comparison of stress intensity factors for a center cracked panel

\begin{tabular}{cccc}
\hline$a / b$ & $\begin{array}{c}\text { Ref. }[13] \\
F(a / b)\end{array}$ & $\begin{array}{c}\text { Calculated } \\
F(a / b)\end{array}$ & $\%$ Error \\
\hline 0.2 & 1.0246 & 0.995 & -2.9 \\
0.4 & 1.1094 & 1.080 & -2.9 \\
0.6 & 1.3033 & 1.279 & -2.4 \\
0.8 & 1.8160 & 1.848 & 1.9 \\
\hline
\end{tabular}

Table 3. Comparison of stress intensity factor for a side cracked panel. The percent error was calculated with respect to results obtained by Cruse and Wilson[14] for the first two rows, and with respect to Bowie[15] for the last two rows

\begin{tabular}{lccccc}
\hline$a / b$ & $\begin{array}{c}\text { Ref. [7] } \\
F(a / b)\end{array}$ & $\begin{array}{c}\text { Ref. [14] } \\
F(a / b)\end{array}$ & $\begin{array}{c}\text { Ref. [15] } \\
F(a / b)\end{array}$ & $\begin{array}{c}\text { Calculated } \\
F(a / b)\end{array}$ & $\%$ Error \\
\hline 0.125 & 1.26 & 1.27 & - & 1.299 & 2.28 \\
0.15 & 1.30 & 1.34 & - & 1.362 & 1.64 \\
0.20 & 1.40 & 1.48 & 1.49 & 1.505 & 1.01 \\
0.30 & 1.67 & 1.82 & 1.85 & 1.867 & 0.92 \\
\hline
\end{tabular}

level of discretization) for values of $(t / a)=-0.5$ and 0.0 is due to having the interacting crack tips closest to each other while at the same time giving the singularity a wider exposure to the stress-free crack surfaces. Both effects imply that the stress field gradients are especially severe for these two cases and hence require a higher degree of discretization or using higher-order elements.

(ii) Four parabolic and two two-parameter crack tip elements were used for each crack. The results are in good agreement with analytic results of Tada et al.[13].

(iii) Five parabolic elements were used for every stress-free surface, three elements were used for the top and bottom surfaces, and four parabolic and two crack tip elements were used for the "center" crack. The results were compared with results by Tada et al.[13]. The calculated results compare well with the above-mentioned reference.

(iv) Five parabolic elements were used for the top and bottom surfaces, as well as for the right stress-free side. Fourteen elements were used for the left stress-free side intersecting the crack, and 14 parabolic and one crack tip element was used for the uniformly pressurized crack. The tip of the element used in discretizing the crack that intersects the free boundary was allowed to have a dislocation extending to infinity to the left, in order not to introduce a dislocation singularity at that intersection point. Good agreement was obtained for the stress intensity factors when compared with several other references (Cleary and Annigeri[7], Cruse and Wilson[14] and Bowie [15]); however, a large number of degrees of freedom ( $262 \mathrm{dof})$ were required to obtain those results.

\section{LINE SPRING MODELLING OF PART-THROUGH CRACK PROBLEMS}

The propagation of part-through cracks in plates using the line spring model has been implemented using this boundary element method.

We first summarize the basic concepts behind the line-spring model first proposed by Rice and Levy[8]. Plates having part-through cracks and loaded by in-plane and moment loads, Fig. 11(a), have "2-D qualities" for the major part of the plate (i.e. the net moment and the thickness averaged stress and displacement fields are sufficient to accurately describe the behavior of the plate). The region of exception is that which is close to the part-through crack where the variation of the stress and displacement field in the thickness direction is large.

To overcome that difficulty, the region around the part-through crack is shrunk onto a single 


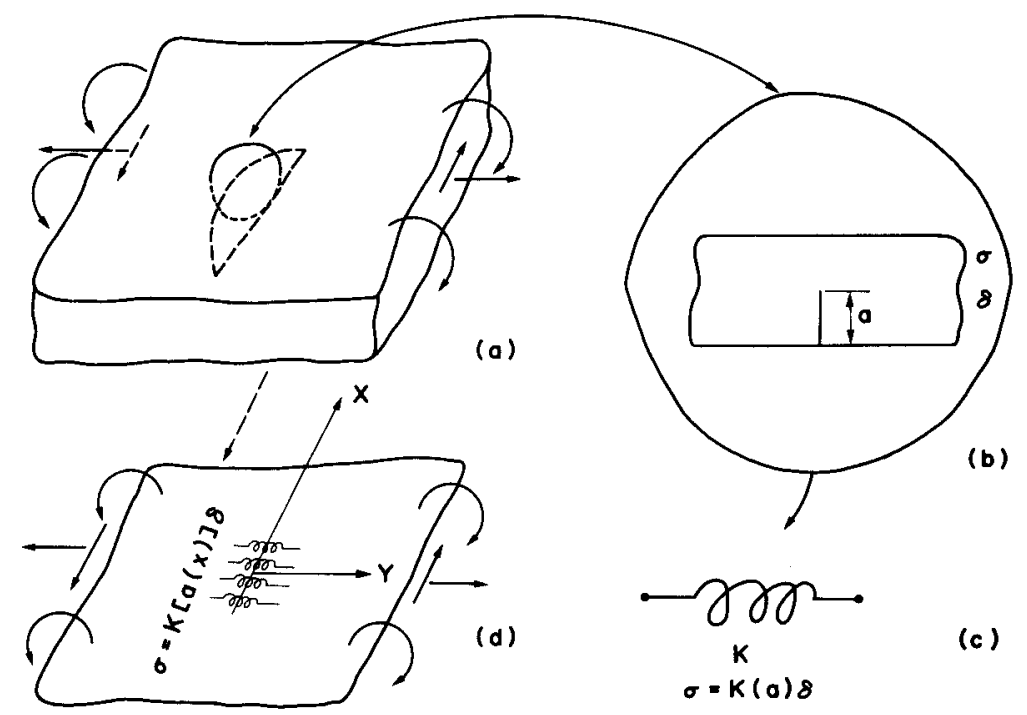

Fig. 11. The construction of the line spring model starting from the 3-D part-through crack geometry (a) in a plate being loaded by in-plane as well as by moment loads. A local cross-sectional thin slice of the partthrough crack (b) is analyzed as a 2-D planar problem which provides a spring relation of thickness averaged stress to displacement (c) used as the boundary conditions in a 2-D line spring model of the 3-D problem (d).

line of discontinuity having a distribution of "springs". Hence the problem is reduced to solving a 2-D problem having a line of discontinuity over which "springs" of appropriate compliances are distributed [Fig. 11(d)]. This is the essence of the "line-spring" modelling procedure. This representation is justified due to the localized deformation field generated by the presence of the partthrough crack.

More specifically, the discontinuity line used to represent the part-through crack in the linespring technique is actually that part of the plate which surrounds the part-through crack and beyond which the stresses and displacements become nearly uniform through the thickness. Due to the high decay rate of the singularities associated with a part-through crack, the region under consideration might be of the order of a few thicknesses away from the part-through crack. Therefore, if the lateral dimensions of the plate are much larger than the thickness, a line spring model is justified, on condition that the discontinuity line transmits compliances that are consistent with the presence of a part-through crack. The determination of the local compliance is carried out by solving the plane strain (for mode I and mode II) or anti-plane (for mode III) edge cracked strips having the 2-D geometries described by taking local cross-sections perpendicular to the plane of the part-through crack being studied, Fig. 11(b), and deriving the thickness averaged traction versus thickness averaged slip relations, i.e. "springs" [Fig. 11(c)].

The thickness averaged traction versus thickness averaged slip relation at any given point along the discontinuity depends on the crack depth. For a fixed geometry of the part-through crack, the relationship between averaged traction versus averaged slip across the discontinuity is linear.

When studying the propagation of part-through cracks, the geometry of the part-through crack changes as the part-through crack grows with increasing load for a given fracture criterion and given fracture toughness. The fracture toughness can in general be inhomogeneous (and the fracture criterion for propagation is in general a nonlinear function of the prevailing local stress field as well as the local material properties and current penetration depth of the crack). The changing crack depth and/or the inhomogeneous fracture toughness leads to nonlinearity in the springs. The method chosen for the solution of the nonlinear equations encountered in the simulation of the propagation of part-through cracks is the secant method. Solutions of some part-through crack propagation problems applied to studying earthquake source processes have been reported by Li and Fares[16]. Some sample results are described below to illustrate the details of the numerical implementation of line spring models and nonlinear spring boundary conditions.

The geometry of the part-through crack is shown in Fig. 12. A through-thickness planar crack of length eight times the plate thickness is flanked by part-through cracks extending twice the distance on both sides. It is assumed that the dominant mode of propagation of the part-through crack would 


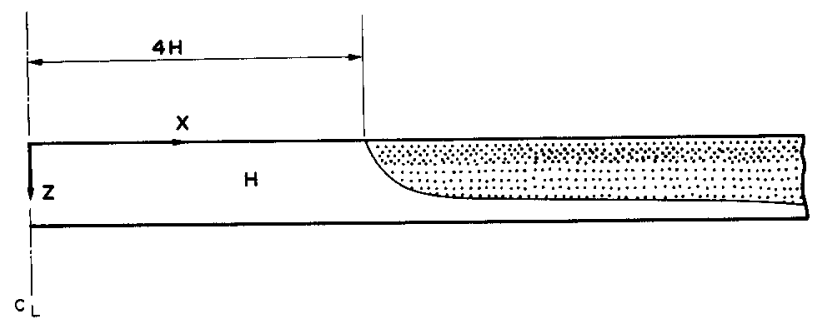

Fig. 12. A part-through crack on either side of a through-thickness crack of length eight times the thickness has to penetrate through a plate having a weak lower layer and a tough upper layer (shaded).

be mode III. In particular, we focus on the effect of the through-thickness crack on the adjacent sections of the part-through cracks. Material toughness (in mode III) varies through the thickness in the form of a Gaussian distribution having the mean at a depth of 0.2 the thickness of the plate from the top surface, and a "spread" of 0.13 the thickness of the plate. This specification of toughness implies a tough upper plate region of thickness approximately 0.4 the thickness and a weak lower plate region. Thus, under constant increasing applied loads, the part-through crack will propagate rapidly initially until it reaches the high toughness region. However, it may be expected that the loadcarrying capacity diminishes as stress concentration forces drive the crack into the tough region, resulting in a / ocally reduced ligament size. This will eventually lead to unstable crack propagation or an instability. It may also be expected that such an instability will initiate in the section immediately adjacent to the through-thickness crack, due to stress transfer effect.

The numerical modelling of this problem is as follows. The through-thickness crack is modelled using nine parabolic elements on which a zero traction boundary condition is enforced. The partthrough cracks are each modelled using five parabolic elements at which the nonlinear spring boundary condition (discussed above) is enforced. Finally, a transition region between the cracked and uncracked region of the plate (i.e. the far tips of the part-through crack) is modelled as a long cohesion zone (of size 16 times the thickness) using one smoothly varying crack tip ( $r^{3 / 2}$ opening) for each tip region. No boundary conditions are imposed on the crack tip elements; however, additional collocation points on the elements adjacent to the crack tip elements are chosen. The effect of this procedure is to cause the dislocation value between the crack tip elements and the adjacent parabolic elements to be nearly continuous. Note that the type of elements used at the tips are implicit boundary conditions. The choice was made so that the stresses are everywhere finite in the plate, and so that the region of interest (i.e. the region adjacent to the through-thickness crack) is not affected by excessive "stiffness" from the tip regions of the part-through crack. Instability here is interpreted as infinite increase in crack depth at any location on the part-through crack for finite increase in applied load at any location on the part-through crack.

The results of this simulation are shown in Fig. 13(a)-(c). The far field shear stress value is increased until instability occurs. The results are calculated at the collocation points only and interpolation is carried out in the plots. Figure 13(a) shows how the propagation through the depth occurs. Note that the through-thickness propagation is steepest in the region that is adjacent to the through-thickness crack (this region will be referred to as the process zone). Figure 13(b) shows the profile of the thickness averaged slip values at different load steps. The thickness averaged slip in the through-thickness crack is nearly an order of magnitude higher than in the part-through cracked region, and joins at a high gradient of slip with the process zone thickness averaged slip values. In Fig. 13(c) the thickness averaged stress profiles at different load values are shown. There is a thickness averaged stress concentration in the process zone. In addition, the stress drops asymptotically to the far field loading value at a distance of a few thicknesses away from the process zone. This indicates that the cohesion zone elements were adequate for the modelling. Finally, note how the stress in the process zone that is closest to the through-thickness crack is undergoing an unloading to the adjacent material at the highest load value which indicates the onset of instability, as indicated earlier.

\section{CONCLUSION AND FINAL REMARKS}

The use of linear elastic dislocation dipole distributions to model cracks and displacement discontinuities leads to a good representation of their stress and displacement fields. Specifically, 

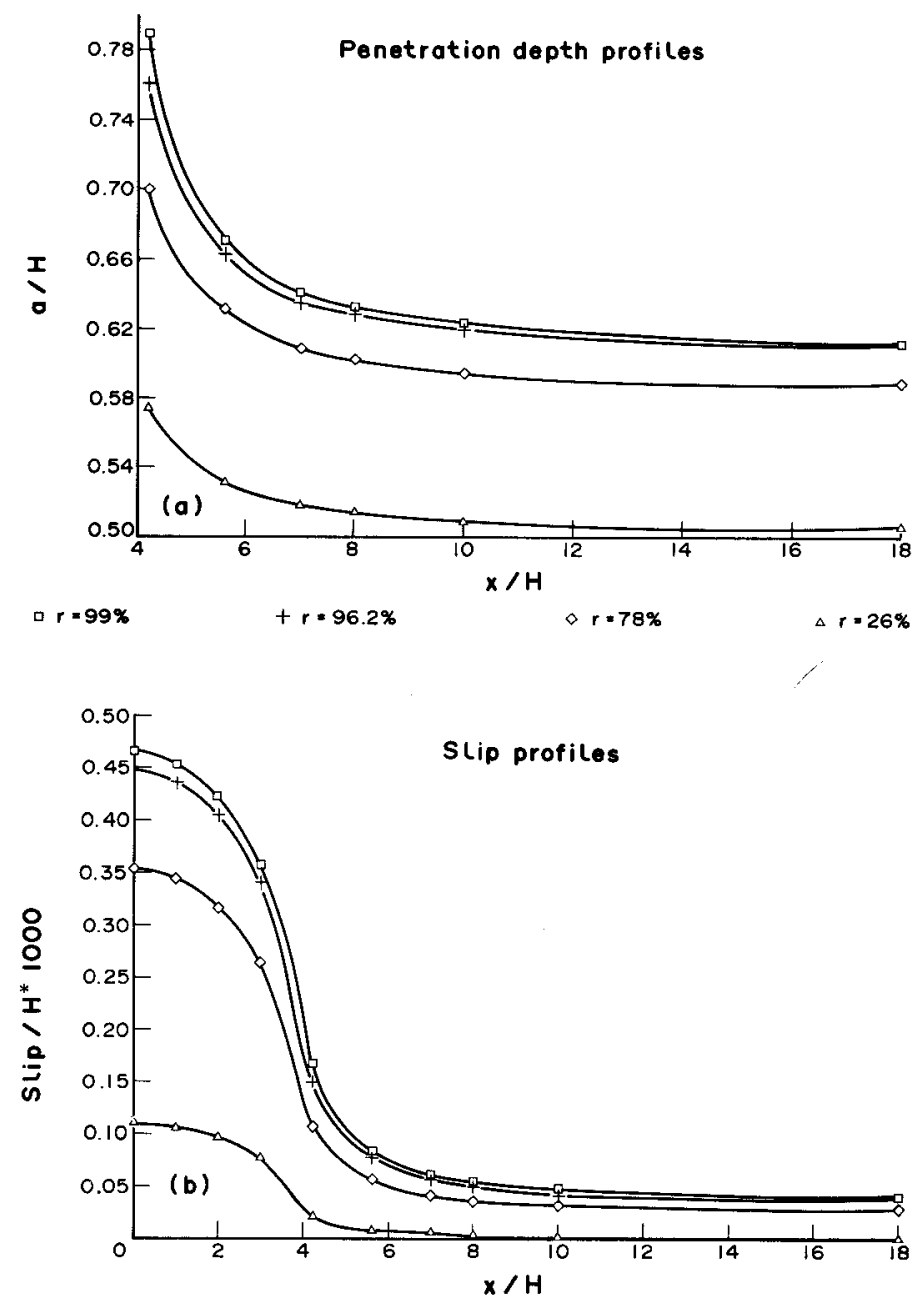

व $r=99 \% \quad+r=96.2 \% \quad \Delta r=78 \% \quad \Delta r=26 \%$

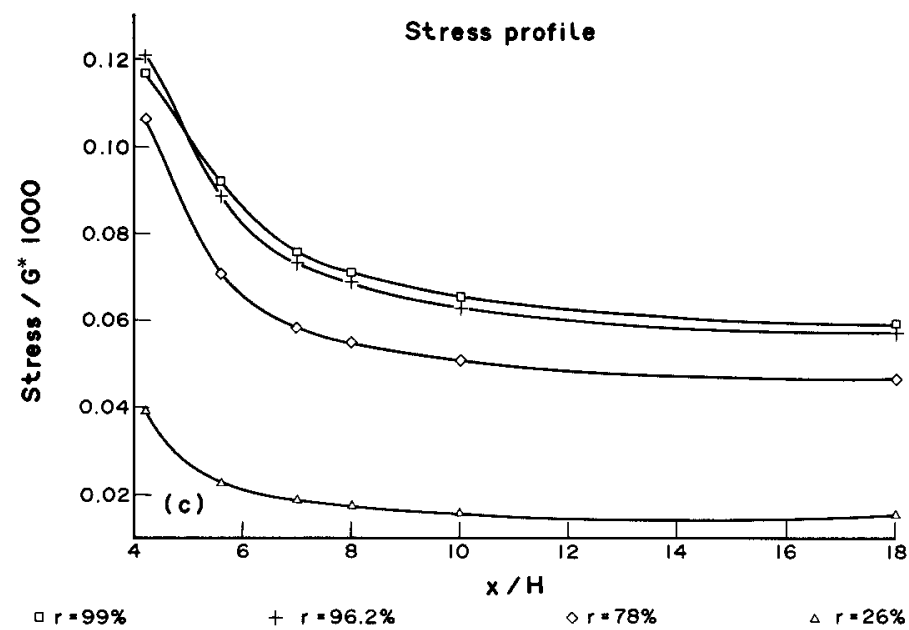

Fig. 13. Results for the simulation of the propagation of the part-through crack shown in Fig. 12. The penetration depth profiles of the part-through crack at different load values (" $r$ " is the ratio of the applied load over the instability causing load) is shown in (a). The part-through crack's penetration is highest in the region closest to the through-thickness crack particularly at loads close to instability. The thickness averaged slip profiles shown resemble the elliptic shapes of sharp cracks except for a trailing process zone at the tips $(x \approx 4 H)$ (b). The thickness averaged stress profiles indicate stress intensification at the zone adjacent to the through-thickness crack (c). At the last load value an unloading of stress is seen to occur in the most penetrated region. 
good results for stress intensity factors could be obtained with a small number of degrees of freedom (i.e. parameters) in the problem. However, care has to be taken in the procedure used for setting up the equations that evaluate the unknown parameters (e.g. collocation, least square fit for the boundary conditions, "zero net average stress along each crack criteria" for stress-free cracks), of which collocation at the Chebyshev points is a general, easy to use and reliable method.

The indirect boundary element method (using the superposition of the dislocation stress fields) leads to inaccurate representation of the solution near the boundaries, and may lead to inefficient or inaccurate solutions when solving for finite bodies with cracks. One method of alleviating this shortcoming is by hybridizing the finite element method (which supplies regularly varying fields) with the boundary element method for the cracks (which supplies the singularities of the problem), as was done by Annigeri and Cleary[7]. Another approach for improving the solution for the stresses near the boundaries using the indirect boundary element method is by using point force based elements for the elements tracing the boundaries and dislocation based elements for the modelling of the discontinuity lines within the region.

Numerical integration of the line integrals leads to a better control over the possible elements that can be used, and several classes of problems can make use of this flexibility, such as multiple Griffith crack problems in infinite space and cohesion zone models.

The indirect boundary element method can readily handle nonlinear boundary condition problems. This allows us to implement a powerful quasi-three-dimensional method known as the line spring method.

Based on the results from this study, it appears worthwhile to extend the present work to include Green's functions which account for some simple boundary conditions implicitly (e.g. half space, circular holes, etc.). This approach is especially valuable when considering 3-D infinite boundaries problem as well as half space problems. The major restriction is that the medium has to be linear and that it can be substructured into piecewise nonhomogeneous regions (this includes linear viscoelastic problems). To extend the use of discontinuity elements as well as the imbedding of analytical results into numerical formulations for nonlinear problems, further research on the hybridization of the finite element method with a general superposition method is required. The elastic fields to be superposed should be independently chosen by the user, and the contribution of those chosen fields to the totality of the solution fields would then be numerically determined.

\section{REFERENCES}

[1] S. L. Crouch, Solution of plane elasticity problems by the displacement discontinuity method. Int. J.numer. Meth. Engng 10, 301-343 (1976).

[2] C. A. Brebbia, The Boundary Element Method for Engineers. John Wiley, New York (1978).

[3] R. Butterfield and P. K. Banerjee, Boundary Element Methods in Engineering Science. McGraw Hill (1981).

[4] A. M. Blandford, A. R. Ingraffea and J. A. Liggett, Automatic 2-D quasi-static and fatigue crack propagation using the boundary element method. Report 81-3, Department of Structural Engineering, Cornell University (January 1981).

[5] A. M. Crawford and J. H. Curran, Higher order functional variation displacement discontinuity elements. Int. J. Rock Mech. Min. Sci. Geomech. Abstr. 19, 143-148 (1982).

[6] J. H. Curran and L. Vandamme, Determination of the 2-D Distribution of Stresses Around a Crack (ISBN 0-7727-7050-6) (July 1983).

[7] B. S. Annigeri and M. P. Cleary, Quasi-Static Fracture Propagation Using the Surface Integral Finite Element Hybrid Method. In Computational Fracture Mechanics-Nonlinear and 3-D Problems. PVP-Vol. 85, AMD-Vol. 81, (Edited by P. D. Hilton and L. N. Gifford), ASME (1984).

[8] J. R. Rice and N. Levy, The part-through surface crack in an elastic plate. J. appl. Mech. 185-194 (March 1972).

[9] M. Kachanov, A simple technique of stress analysis in elastic solids with many cracks. Int. J. Fracture 28, R11-R 19 (1985).

[10] G. I. Barenblatt, Advances in Applied Mechanics (Edited by H. L. Dryden and T. Karman), Vol. 7, pp. 56-131. Academic, New York (1962).

[11] S. P. Timoshenko and D. H. Young, Elements of Strength of Materials. Van Nostrand Reinhold, New York (1968).

[12] T. M. Yokobori, Uozumi and M. Ichikawa, Interaction between non-coplanar parallel staggered elastic cracks. Rep. Conf. Inst. Strength and Fracture of Materials 7, 25 47, Tohoku University (1971).

[13] H. Tada, P. C. Paris and G. R. Irwin, The Stress Analysis of Cracks Handbook. Del Research Corp., Hellertown, PA (1973).

[14] T. A. Cruse and R. B. Wilson, Advanced applications of boundary integral equation methods. Nuclear Engng Des. 46, 223-234 (1978).

[15] O. L. Bowie, Solutions of plane problems by mapping techniques, in Methods of Analysis and Solution of Crack Problems (Edited by G. C. Sih). Noordhoff (1973).

[16] V. C. Li and N. Fares, Rupture Processes in the Presence of Creep Zones, in Earthquake Source Mechanics. Ewing Series V.6, (Edited by S. Das et al.), pp 71-80 (1986).

(Received 9 January 1986) 